\section{Topics in bioinorganic chemistry}

Techniques and Topics in Bioinorganic Chemistry. (Aspects of Inorganic Chemistry.) Edited by C. A. McAuliffe. Pp. $\mathrm{xv}+351$. (Macmillan: London and Basingstoke; February 1975.) $£ 20.00$.

THE frontier between inorganic chemistry and biochemistry has been crossed so often that the border is now illdefined. It is too early to know how the buffer state, which bioinorganic chemistry or inorganic biochemistry may be considered to constitute, will evolve. Certainly, the subject is fashionable. But that which is fashionable is not necessarily respectable, the latter description, evocative of safe, solid virtues, being more often applied to the mainstreams of the parent subjects. But irrespective of prejudices it is impossible to ignore the importance of those elements which normally constitute the province of inorganic chemists, to biological processes. The roles of proteins containing iron and copper in all manners of redox reactions and oxygen transport are well known, and the extent to which other ions, such as zinc, are involved is slowly being revealed. Many other elements, for example, selenium, are known or suspected to be required but their functions ill-defined.

This book is one of several which have appeared recently and which seek to attract attention to the current excitement for the subject. In the first chapter M. W. Makinen attempts ambitiously to relate the functions of some proteins to structural details derived from X-ray diffraction studies occasionally reinforced by information gleaned from spectroscopic investigations. J. M. Pratt, in a chapter entitled "Principles of Catalysis by Metalloenzymes", considers the haemoglobins and myoglobins, catalase and peroxidase, and nitrogenase. The biochemical function of molybdenum is described by F. L. Bowden in a well planned review. Since the environments of molybdenum in proteins such as xanthine oxidase and nitrogenase are unknown, speculation about the role of molybdenum in these proteins is subdued. Some interesting chemistry of molybdenum is, however, included; the relevance to the biochemical function is not overstated. $\mathbf{J}$. Webb discusses a number of complexes and proteins involved in iron transport and storage, with considerable emphasis on the electronic structures of the interacting iron ions. The volume is completed by an all-toobrief introduction by S. J. Ferguson to the use of nuclear magnetic resonance spectroscopy in the investigation of the structures of proteins in solution.

Books containing chapters by different authors often suffer from two defects. First, the delay between completion of the manuscripts and publication leads, in a rapidly developing subject, to redundant speculation. Second, although the editor in his introduction seeks to justfy overlap between the contents of the various chapters, this may prove irritating to those prepared to read through the book. For example, the binding of molecular oxygen to iron in myoglobin and haemoglobin is considered at length in chapters 1 and 2 , as is nitrogen fixation in chapters 2 and 3 . But as it is likely that the volume will be used as a source of review articles, this criticism may not be pertinent.

H. A. O. Hill

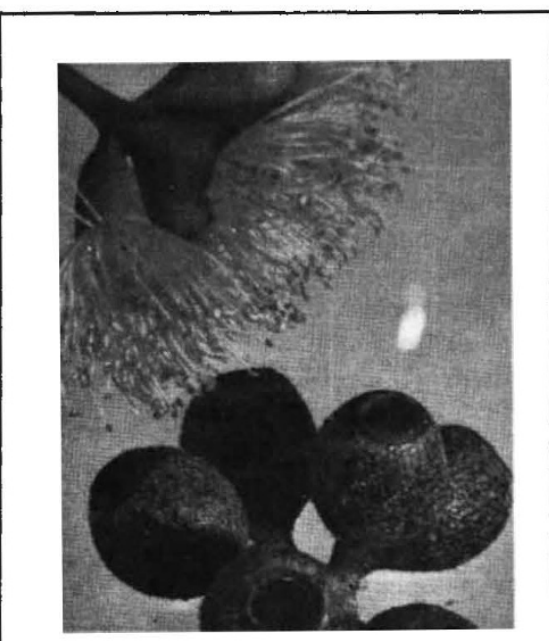

Eucalyptus jucunda C. A. Gardner, usually $2-3.5 \mathrm{~m}$, but sometimes up to $8 \mathrm{~m}$ high. Photo shows stamens and elliptical fruiting bodies $(1.5 \times 1.2 \mathrm{~cm})$. Taken from Eucalypts of the Western Australia Goldfields by G. M. Chippendale. Pp. iv +216 . (Australian Government Publishing Service: Canberra, 1973.) A $\$ 3.75$.

\section{Casting light on structures}

Biochemical Spectroscopy. By Richard Alan Morton. Vol. 1: pp. xvi+1-381; vol. 2: pp. 382-873. (Adam Hilger: London, March 1975.) $£ 80.00$ for set.

THE title Biochemical Spectroscopy may today conjure up an image of a work dealing fairly exhaustively with the practical and theoretical aspects of the spectroscopy, primarily, of current molecular biology. It might, for instance, emphasise the applications in that field of the more modern techniques of ESR, Mössbauer and NMR, and perhaps give as much weight to circular dichroism, optical rotatory dispersion and Raman as to the older basic techniques.

This book is, however, of a quite different kind. Essentially, it consists of a large number of monographs, grouped rather arbitrarily into longer sections. Of these, some, like those entitled "Water and Sunlight in a Biological Context' and 'Electronic Absorption Spectra', give background material of a relatively generalised kind but most of them are groups of monographs, many quite short, on classes of natural products and the light that spectroscopic methods have cast (and in a few cases might yet cast) on their structures, functions and interrelations. The range of topics is wide, and it is revealing to see in how many and diverse fields the author has himself made contributions in a laboratory career lasting 50 years. A chemist with no special interest in spectroscopy could do worse than to turn first to this book if he should have to deal with a natural product belonging to a known but unfamiliar class; the treatment of the chemistry is always interesting, if necessarily incomplete, and the references are excellent. The writing is clear and straightforward, and, though the bias is towards classical visible and ultraviolet spectroscopy, developments based on other techniques are not neglected.

In places the reader encounters unexpected hurdles, however. Some of these arise from a failure to number, and thus to correlate with the text, the numerous structural formulae embedded in it, and in places the lack of either names or reference numbers, coupled with a sprinkling of errors, makes the going hard indeed for a reader unfamiliar with the field. The sources of much of the tabulated data are not given, and some sections wear the antique look which is conveyed by the bulgy contours of 30 or 40 year-old spectral diagrams; these, however, normally occur where the more modern data which might otherwise supersede them are incomplete. Athough in places some updating of material is surely needed, a prime defect is the plethora of small errors and misprints which defaces this handsomely produced-and extremely expensive-pair of volumes.

The price is such, in fact, that the volumes can only be considered as a library reference work. This is a great pity, since a deal of pleasure and information is to be had from random browsing. It is, however, clearly impossible to suggest cuts and economies in presentation which could result in a price reduction by the order of magnitude which is needed to make the volumes as accessible as they deserve to be.

E. A. Johnson 